\title{
Microwave-assisted Preparation of Amino Modification Montmorillonite and the Capturing Performance of Carbon Dioxide
}

\author{
$\mathrm{Na} \mathrm{Li}{ }^{1, \mathrm{a}^{*}}$, Zhebin $\mathrm{Yu}^{1, \mathrm{~b}}$ and Zhanghao Chen ${ }^{1, \mathrm{c}}$ \\ ${ }^{1}$ College of resources and materials, Northeastern university at Qinhuangdao, Qinhuangdao, \\ Hebei Province, China \\ allina_2481@163.com, b405252450@qq.com, ${ }^{c} 1934424474 @ q q . c o m$
}

\begin{abstract}
Keywords: mesoporous, montmorillonite, amino modification, carbon dioxide capture
\end{abstract}
Abstract. The amino modification montmorillonite was prepared using the cetyl trimethyl ammonium bromide as modifier by the microwave irradiation and common heating method. According to the analysis of infrared spectra, scanning electron microscope and specific surface area, it is found that the larger amount of amino modifier has entered effectively into the layer of montmorillonite under the condition of microwave irradiation, so it has the bigger pore size, the smaller particle, more obvious layered structure and the thinner layer thickness. At the same time, according to the capture example of carbon dioxide, it is found that the capture amount of microwave amino modification montmorillonite is the biggest, and it is $150 \mathrm{mg} / \mathrm{g}$.

\section{Introduction}

The global warming resulted from the great emission of carbon dioxide seriously threatens the earthly environment. "Greenhouse Gas Control" has pointed out that, till 2050, carbon dioxide captured globally can reach 2360 billion tons. That means emission reducing potential of carbon capture and storage will be a third of the global total carbon emissions. At present, there are many research reports about capturing material of carbon dioxide, but the cost and energy consumption of this technique is still higher ${ }^{[1-3]}$. However, mesoporous modification montmorillonite gains a lot of attention in the environment pollution control due to its special advantages like cheap and easily accessible, stable properties, developed pores and good adsorption ability. In recent years, a large amount of researches have been conducted at home and outside. And these results all prove that, compared with the original montmorillonite, the adsorption of modification montmorillonite to various pollutants in the water has been greatly improved ${ }^{[4-7]}$. And the study of its application in carbon dioxide capture technology has rarely been reported.

In this paper, the mesoporous amido modification montmorillonite is prepared using microwave heating method. And its capturing ability of carbon dioxide is experimented, and the original montmorillonite and the montmorillonite prepared by common heating method are used as a contrast experiment to provide strong theoretical support for its application of carbon dioxide capture.

\section{Materials and methods}

Preparation of sodium modified montmorillonite The commercial calcium montmorillonite (Ca-Mt) used in the present study was supplied by Tianhong Mining Company, Ltd. (Inner Mongolia China). It was denoted as R1. The cation exchange capacity (CEC) of montmorillonite is 115 $\mathrm{mmol} / 100 \mathrm{~g}$. The basal spacing of $\mathrm{Ca}-\mathrm{Mt}$ is $15.38 \AA$. Na-montmorillonite (Na-Mt) was prepared from Ca-Mt before the organic modification. $100 \mathrm{~g}$ original montmorillonite, $5 \mathrm{~g} \mathrm{Na}_{2} \mathrm{CO}_{3}$ and $900 \mathrm{ml}$ water were mixed under mechanical stirring for $20 \mathrm{~min}$ with the speed of $350 \mathrm{r} / \mathrm{min}$. Then the mixture was put in the microwave over to heat $4 \mathrm{~min}$ under the power of $560 \mathrm{w}$. Subsequently, the obtained mixture was centrifugated, dried and grinded through 100 meshes to get microwave sodium montmorillonite.

Preparation of amino modified montmorillonite The microwave sodium montmorillonite and water were mixed with 1:20 (mg/L) ratio. And then $5 \mathrm{~g}$ amino modifier (cetyl trimethyl ammonium bromide) were added under mechanical stirring for $30 \mathrm{~min}$ to thoroughly mix. Some of the mixture 
were heated for $2 \mathrm{~h}$ in $80{ }^{\circ} \mathrm{C}$ water bath and some others were put in microwave oven to irradiation 10 min under the power of $560 \mathrm{w}$. Subsequently, the obtained mixture was centrifugated, dried and grinded through 100 meshes to get microwave amindo montmorillonite. And they were denoted as R2 and R3, respectively.

Characterization The infrared spectra of montmorillonite were determined by FTIR-8400S - type Fourier infrared spectrometer, which was produced by SHIMADZU company in Japan. The microstructure were obvioused by SUPRA55 SAPPHIRE type scanning electron microscope (SEM), which was produced by Carl Zeiss Company in German. And the pore size and specific surface area were analyzed by SSA-4300 pore size and specific surface area analyzer, which was produced by Beijing Builder Electronic Technology Company.

Experiment of carbon dioxide capturing capability Carbon dioxide capture experimental apparatus was shown in Figure 1. The mesoporous modified montmorillonite prepared by different methods were placed in the adsorbent bed at $70{ }^{\circ} \mathrm{C}$ and the stable flow rate. In a certain period of time, the quality of carbon dioxide was measured before and after the adsorption bed, and the difference between them was the capture of carbon dioxide.

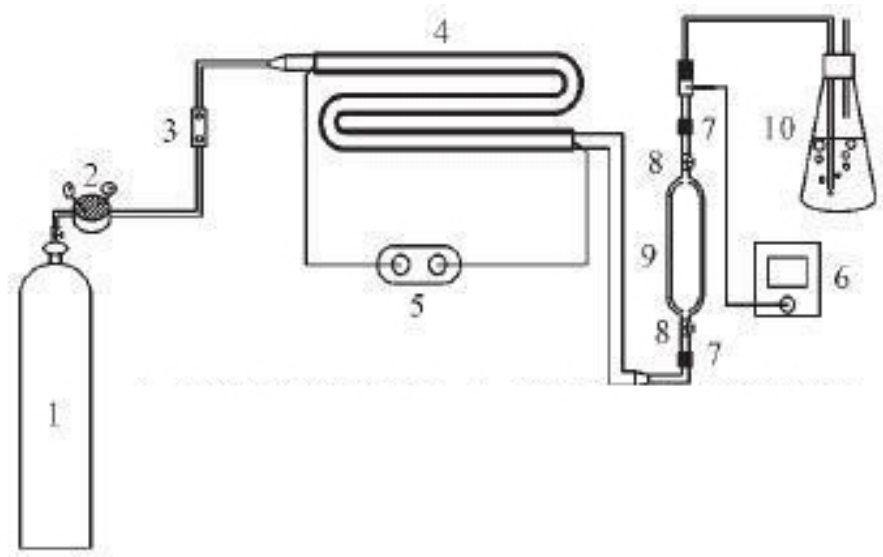

Fig. 1 Carbon dioxide capture experimental apparatus

1 gas cylinder of carbon dioxide 2 pressure reducing valve 3 flowrator 4 Electric heating belt 5 thermocouple 6 temperature indicator 7 mass flowmeter 8 connect 9 adsorption bed 10 Tail gas absorption bottle

\section{Result and Discussion}

FT - IR spectrum analysis An infrared spectrum analysis of the montmorillonite and two kinds of amino modified montmorillonite were carried on, and the results were showed in the figure 2.In the diagram, the bigger absorption peak at $1031 \mathrm{~cm}^{-1}$ is the stretching vibration peak of $\mathrm{Si}-\mathrm{O}-\mathrm{Si}$, which is the characteristic absorption peaks of the montmorillonite. It can be seen that after the modification, this peak does not change. So the basic structure of montmorillonite does not destroy.

However after organic modification, the characteristic absorption peaks of the quaternary ammonium salt cationic at $2916 \mathrm{~cm}^{-1}, \mathrm{C}-\mathrm{H}$ bond stretching vibration peak at $2848 \mathrm{~cm}^{-1}$ and $\mathrm{C}-\mathrm{N}$ bond bending vibration absorption peak at $1487 \mathrm{~cm}^{-1}$ are found. This made it clear that whatever the modification method is used, the long carbon chain quaternary ammonium salt cations can be effectively into the interlayer structure of montmorillonite. Comparison of the peak intensity of these wave numbers, it is found that the intensity of the new peak of the modified montmorillonite using microwave irradiation is more than that of the conventional heating method. This may be due to the comparison with the conventional heating method, under the condition of microwave irradiation, the molecules of montmorillonite and cetyl trimethyl ammonium bromide obtain the higher energy and apace collide, so the reaction of ion exchange is more easily happened. 
The absorption peak at $3400 \sim 3650 \mathrm{~cm}^{-1}$ is the characteristic absorption peaks of $-\mathrm{OH}$. After the modification, the strength of the peak has an obvious weakening trend. It is because the addition of amino modifier has the hydrophobic effect, which cause the smaller association between the hydroxyl.

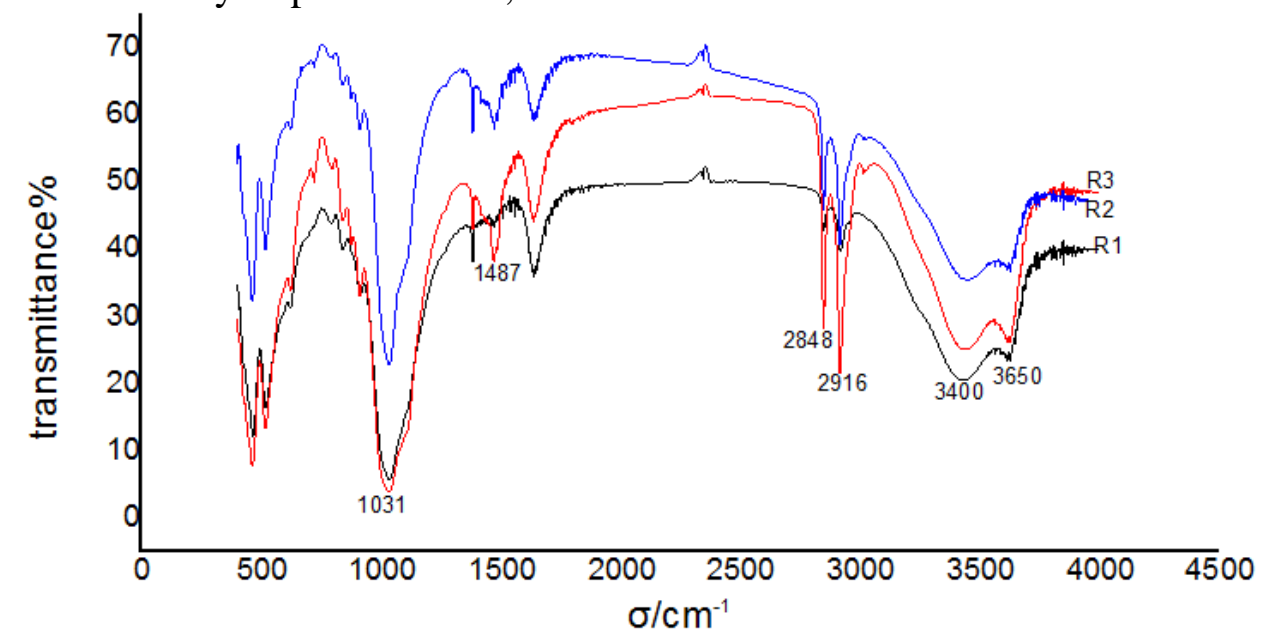

Fig. 2 infrared spectra of three kinds of materials

Results of SEM The SEM analysis of three kinds of montmorillonite are carried on, and the results are shown in Figure 3, Figure 4 and Figure 5 ( $\times 50,000$ times). It can be seen in the figures, surface thickness of the original montmorillonite varies, granular lumps aggregate, particle surface extremely is not flat, and the edge is not smooth. However, after the organic modification, there are small particles, even distribution, obvious layered structure, thin layer thickness and more gaps.
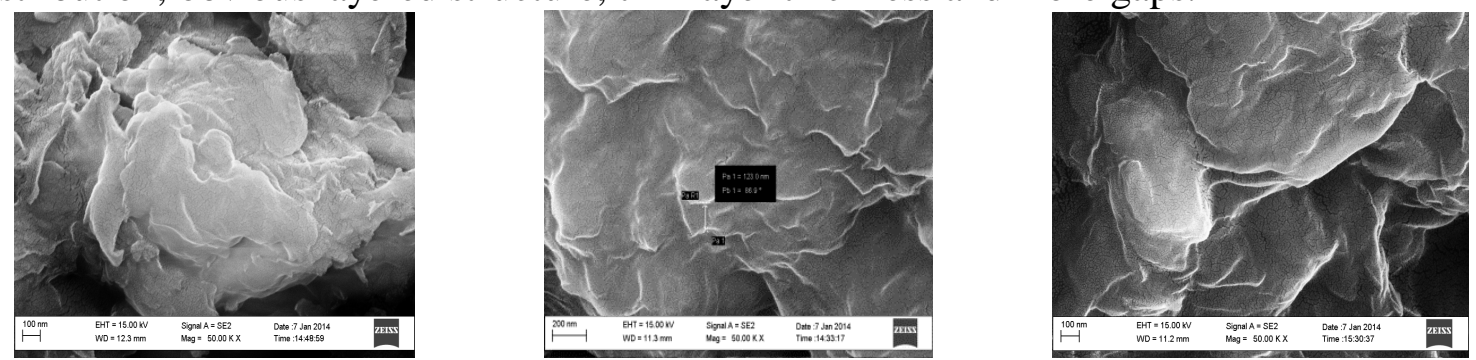

Fig. 3 SEM image of simple R1 Fig. 4 SEM image of simple R2 Fig. 5 SEM image of simple R3

Specific surface area and pore size analysis The specific surface area and pore size of three kinds of montmorillonite are analyzed, and the results are shown in the Table 1. As can be seen from Table 1, specific surface area of the modified montmorillonite reduces significantly, while the single-point average pore size increases. The reason may be that the cationic of quaternary ammonium enters the montmorillonite, which cause the bigger pore. Compared to the microwave and common heating method, the specific surface area and pore size has increased. It possibly due to the more amino modifier entering into the layer of montmorillonite. Therefore, microwave modification montmorillonite has the more pore, the bigger pore size and specific surface area.

Table 1 surface area and pore size data of three kinds of montmorillonite

\begin{tabular}{cccc}
\hline Simple & BET specific surface area $\mathrm{m}^{2} / \mathrm{g}$ & Total pore volume cc/g & $\begin{array}{c}\text { Single-point average pore } \\
\text { radius /A }\end{array}$ \\
\hline R1 & 25.738 & 0.139 & 108.6 \\
R2 & 6.078 & 0.074 & 245.4 \\
R3 & 8.51 & 0.124 & 290.3 \\
\hline
\end{tabular}

Capturing performance of carbon dioxide The capturing experiments of carbon dioxide on three kinds of montmorillonite are carried on under the condition of $70{ }^{\circ} \mathrm{C}$ and $0.2 \mathrm{~L} / \mathrm{min}$, respectively. The amount of captured $\mathrm{CO}_{2}$ per gram of montmorillonite is calculated, and the results are shown in Table 2.

From Table 2 , It can be seen that compared with montmorillonite, the capturing quantity of carbon dioxide has been greatly improved on organic modified montmorillonite. At the same time, the capturing performance of carbon dioxide on the microwave modified montmorillonite is better than 
that on the common modified montmorillonite. The capturing quantity of carbon dioxide can reach 150 $\mathrm{mg} / \mathrm{g}$.

Table 2 Capture quantity of carbon dioxide on three kinds of montmorillonite

\begin{tabular}{cc}
\hline Simple & Capture quantity of carbon dioxide $(\mathrm{mg} / \mathrm{g})$ \\
\hline R1 & 100 \\
R2 & 124 \\
R3 & 150 \\
\hline
\end{tabular}

Combined with the results of characterization, under the condition of microwave irradiation, the modification montmorillonite has the smaller particles, more obvious layered structure, the thinner layer thickness, more space, bigger pore size and more amino modifier. These may cause that the carbon dioxide enters the montmorillonite easily, so it is more suitable for the adsorption of carbon dioxide on its surface. At the same time, due to entering a large number of quaternary ammonium salt, the carbon dioxide may react with the amine ion, therefore, the capturing performance of $\mathrm{CO}_{2}$ is increased.

\section{Conclusion}

Modified montmorillonite has not changed the inherent structure of montmorillonite, but the quaternary ammonium salt cationic has been inserted between the layers. The pore size of microwave modification montmorillonite is $290.3 \mathrm{~A}$, and specific surface area is $8.51 \mathrm{~m}^{2} / \mathrm{g}$. After Microwave modification, montmorillonite has the smaller particle, more obvious layered structure, the thinner layer thickness and more. Space. Capture experiments showed that organic modified montmorillonite compares with the original soil on the capture quantity of carbon dioxide has greatly improved. At the same time, the microwave modified montmorillonite on carbon dioxide capturing performance is better than common modified montmorillonite.

\section{Acknowledgement}

This paper was supported by the Fundamental Research Funds for the Central Universities, China (N120423004) and School science and technology support program of Northeastern University at Qinhuangdao, ChinaZ(XNK201409).

\section{References}

[1] W. Zhang, M. Zhang. The $\mathrm{CO}_{2}$ capture technology trend analysis based on the patent and literature.Science and technology management research. 05(2014) 23-27

[2] X. Chen. Carbon capture of ideal and reality. The global business.

[3] J. Zhang. Carbon capture and storage (CCS) development prospects. Renewable resources and recycling economy. 06(2013)12-17.

[4] L. X. Jang, N. Liu, S. Q. Zhao, S. Chang. The adsorption study of modified montmorillonite on the Congo red. Mineral and chemical processing. 02(2015)8-12

[5] D. H. Gao, X. J. Lv, J. Z. Ma.The research progress of modified montmorillonite on the properties of chromium ion. Chinese leather.43(2014)17-21

[6] H. Wang, X. Chen, Z. L. Du. The adsorption performance study on cationic modified montmorillonite to reactive turquoise blue K - GL dye. Chemical research and application. 26(2014)7-12

[7] A. Azzouz, E. Assaad, A. V. Ursu, T. Sajin, D. Nistor, R. Roy. Carbon dioxide retention over montmorillonite-dendrimer materials. Applied Clay Science, 48(2010) 133-137 